\section{RGM et néogénine: un jeune couple prometteur}

Eiji Matsunaga, Alain Chédotal
Équipe Développement

neuronal, CNRS UMR7102,

Université Paris 6,

9, quai Saint-Bernard,

75005 Paris, France.

chedotal@infobiogen.fr

treint pas au seul guidage axonal. Pour mieux connaître le rôle précoce de ces molécules, nous avons choisi de perturber leur expression normale dans le système nerveux en utilisant la technique d'électroporation in ovo [3].

Cette méthode mise au point au Japon permet de faire entrer sous l'effet d'impulsions électriques de l'ADN dans les cellules en division, notamment dans le système nerveux d'embryon de poulet. Nous avons employé différents vecteurs permettant soit d'augmenter l'expression de la néogénine ou de RGM, soit, au contraire, de les faire disparaître. Cette dernière approche fait appel à la technique des petits ARN interférents. Nous avons constaté que la surexpression de la néogénine, mais pas celle de RGM, provoquait une forte élévation de la mort cellulaire apoptotique dans le système nerveux [3]. Ce résultat rappelait ceux obtenus par le groupe de Patrick Mehlen (CNRS, Lyon, France) avec DCC (deleted in colorectal cancer) un récepteur de la nétrine-l de structure moléculaire proche de la néo-

\section{génine $(\rightarrow)$.}

$\varepsilon n$ effet, diverses études indiquent que $D C C$ appartient à la famille des $(\rightarrow) \mathrm{m} / \mathrm{s}$ 2001, n०6-7, p. 744 et 2003, $n^{\circ} 11$, p. 1062 récepteurs dits à dépendance: quand leur ligand est présent, ces récepteurs agissent positivement sur les cellules qui les expriment, par exemple en stimulant la croissance axonale dans le cas de DCC et de la nétrine-1. En l'absence de ligand, le récepteur n'est pas inactif, mais induit la mort des cellules qui l'expri- 
ment. En collaboration avec l'équipe de P. Mehlen, nous avons donc cherché à savoir si la néogénine était un récepteur à dépendance pour RGM. Nous avons montré que, si l'on diminue l'expression de RGM avec des ARN interférents, on peut stimuler la mort cellulaire dans le système nerveux dans des proportions équivalentes à celle de la néogénine. Cet effet pro-apoptotique est dépendant de la présence de néogénine car il est bloqué si on supprime simultanément la néogénine et RGM. L'utilisation de lignées cellulaires a permis de montrer que la mort induite par la néogénine requiert un clivage de son domaine intracellulaire et l'activation de la voie des caspases. Ces résultats montrent que la néogénine est un récepteur à dépendance et que le rôle des molécules dites de guidage axonal dépasse leur stricte fonction de guidage et qu'elles ont une implication plus large dans de nombreuses étapes du développement du système nerveux. En outre, un nombre croissant de travaux suggèrent que ces molécules sont aussi impliquées dans des processus pathologiques dont certains cancers. À ce titre, il vient d'être montré que RGMC est impliqué dans I'hémochromatose juvénile [4], ce qui laisse présager d'autres surprises avec ces molécules. $\diamond$

RGM and neogenin, a promising couple

\section{REMERCIEMENTS}

Ce projet est soutenu par la Fondation Retina France.

\section{RÉFÉRENCES}

1. Monnier PP, Sierra A, Macchi P, et al. RGM is a repulsive guidance molecule for retinal axons. Nature 2002; 419: 392-5.

2. Matsunaga $\varepsilon$, Tauszig-Delamasure S, Monnier PP, et al. RGM and its receptor neogenin regulate neuronal survival. Nat Cell Biol 2004; 6: 749-55.

3. Rajagopalan S, Deitinghoff L, Davis D, et al. Neogenin mediates the action of repulsive guidance molecule. Nat Cell Biol 2004; 6: 756-62.

4. Papanikolaou G, Samuels ME, Ludwig EH, et al. Mutations in HFE2 cause iron overload in chromosome lq-linked juvenile hemochromatosis. Nat Genet 2004; 36: 77-82.

\section{NOUVELLE}

\section{La huntingtine stimule le transport du BDNF}

Sandrine Humbert, Frédéric Saudou

> L'expansion anormale de glutamines (polyp) dans la protéine huntingtine est à l'origine de la maladie de Huntington, qui affecte plus de 6000 personnes en France. Cette maladie est caractérisée par des mouvements incontrôlés (chorée) et des désordres psychiques et intellectuels conduisant à une incapacité totale, à la démence et, finalement, à la mort des patients. La lésion neuropathologique dans la maladie de Huntington implique le dysfonctionnement et la dégénérescence de certains neurones du cerveau, en particulier des neurones du striatum, une structure impliquée dans le contrôle du mouvement. Malgré de nombreuses études, la fonction de la huntingtine normale ainsi que les mécanismes moléculaires par lesquels la huntingtine polyp (mutante, contenant l'expansion anormale) conduit au dysfonctionnement et à la mort neuronale ne sont pas encore bien compris [1]. Les travaux de L.R. Gauthier et al. [2] apportent de nouveaux élé- ments pour comprendre ces mécanismes et décrivent pour la première fois une fonction pour la huntingtine dans le transport de vésicules contenant un facteur neurotrophique, le brain-derived neurotrophic factor (BDNF). En situation pathologique, cette fonction est altérée, ce qui conduit à la dégénérescence des neurones du striatum.

\section{Le BDNF, un facteur neurotrophique d'importance dans la maladie de Huntington}

Le BDNF est produit par les neurones du cortex et délivré vers le striatum où il est nécessaire à la différenciation et à la survie des neurones de cette région. Pourquoi étudier le BDNF dans la maladie de Huntington? In vitro, l'ajout de BDNF inhibe la mort des neurones striataux induite par la huntingtine polyp. In vivo, les concentrations de BDNF sont dimi- nuées dans le cerveau de patients atteints. Cette diminution de BDNF a d'abord été attribuée à un défaut transcriptionnel [3], mais l'étude de L.R. Gauthier et al. [2] montre qu'elle est également due à un dérèglement du transport vésiculaire et, en conséquence, à un défaut de sa libération dans la synapse.

\section{La huntingtine stimule le transport vésiculaire du BDNF} le long des microtubules

Bien qu'on puisse l'observer dans les noyaux, la huntingtine est majoritairement cytoplasmique, associée à des structures vésiculaires et aux microtubules (Figure 1). Les auteurs [2] ont formulé I'hypothèse selon laquelle la huntingtine pourrait influencer le transport intracellulaire du BDNF dans les neurones du cortex (neurones qui produisent le BDNF et le transportent vers le striatum). Pour étudier la dynamique du BDNF, une technique de vidéomicroscopie rapide 